\title{
Encyclopedia of Sustainability Science and Technology
}

\author{
Living Edition \\ | Editors: Robert A. Meyers
}

\section{Parabolic Trough Solar Technology}

- Eckhard Lüpfert (1) Email author (eckhard.luepfert@dlr.de)

1. Institute of Solar Research, DLR Deutsches Zentrum für Luft- und Raumfahrt, German Aerospace Center, , Cologne, Germany

Living reference work entry

First Online: 31 January 2021

DOI: https://doi.org/10.1007/978-1-4939-2493-6_678-3

- 3 Downloads

\section{Glossary}

Aperture width

Straight distance between mirror edges across the PTC

Absorber tube

Dark tube positioned in the focal line, typically from steel with coating and with evacuated glass envelope to minimize heat loss, converting solar radiation energy into thermal energy in the fluid, see receiver

Collector loop (PTC)

Unit of several PTC connected in series to heat the fluid from inlet to outlet header temperature

Direct normal irradiance, beam irradiance

Direct part of the sunlight, coming from within the sun disk as almost parallel light onto a surface, measured as power density in $\mathrm{kW} / \mathrm{m}^{2}$

Drive (PTC)

Unit consisting of motor and gear or hydraulic drive with valves and cylinders, and the controller to turn the PTC into the correct operational tracking angle 
Ratio of useful energy and total energy input

Efficiency (PTC)

Ratio of thermal energy output from the PTC and total solar radiation received on the aperture area

Heat transfer fluid ("HTF")

Fluid receiving the...

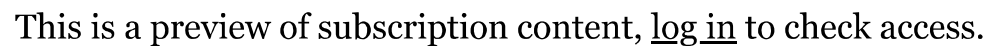

\section{Bibliography}

\section{Primary Literature}

1. Epp B (2019) www.solar-payback.com (http://www.solar-payback.com), www.solarthermalworld.org (http://www.solarthermalworld.org) (solar heat in industrial processes, suppliers, applications)

2. Fernández-García A, Sutter F, et al. (2018) Parameters and method to evaluate the reflectance properties of reflector materials for concentrating solar power technology 3.o. SolarPaces Guideline. http://www.solarpaces.org/csp-researchtasks/task-annexes-iea/task-iii-solar-technology-and-advancedapplications/reflectance-measurement-guidelines/ (http://www.solarpaces.org/csp-research-tasks/task-annexes-iea/task-iiisolar-technology-and-advanced-applications/reflectance-measurementguidelines/)

3. Fredriksson JR (2019) Concentrating solar power: a comparison and evaluation of innovative parabolic trough collector concepts for large scale application Annex B, DLR/TH Wildau. https://elib.dlr.de/130864 (https://elib.dlr.de/130864)

4. Happich C, Janotte N, Pernpeintner J, Schiricke B, Lüpfert E (2018) Second generation linear focus sun simulator to test optical performance of parabolic trough receivers - OptiRec. Solarpaces 2017, AIP conference proceedings 2033, https://doi.org/10.1063/1.5067023 (https://doi.org/10.1063/1.5067023)

5. Hilgert C, Jung C, Wasserfuhr C, Leon J, Valenzuela L (2019) Qualification of silicone based HTF for parabolic trough collector applications. AIP Conference Proceedings 2126:080003. https://doi.org/10.1063/1.5117598

(https://doi.org/10.1063/1.5117598)

CrossRef (https://doi.org/10.1063/1.5117598)

Google Scholar (http://scholar.google.com/scholar_lookup?

title=Qualification\%20of\%20silicone\%20based\%20HTF\%2ofor\%2oparabolic\% 2otrough\%2Ocollector\%2Oapplications\&author=C.\%2OHilgert\&author=C.\%2O Jung\&author=C.\%20Wasserfuhr\&author=J.\%20Leon\&author=L.\%20Valenzue la\&journal=AIP\%20Conference $\% 20$ Proceedings\&volume $=2126 \&$ pages $=08000$ $3 \&$ publication_year $=2019 \& d o i=10.1063 \% 2 \mathrm{~F} 1.5117598)$ 
6. International Electrotechnical Commission (2020) Solar thermal electric plants www.iec.ch/tc117 (http://www.iec.ch/tc117)

Google Scholar (http://scholar.google.com/scholar_lookup?

title=Solar\%20thermal\%2oelectric\%2oplants\&publication_year=2020)

7. Janotte N, Luepfert E, Pottler K, Schmitz M (2017) Full parabolic trough qualification from prototype to demonstration loop, Solarpaces 2016. AIP Conference Proceedings 1850:020010. https://doi.org/10.1063/1.4984334 (https://doi.org/10.1063/1.4984334)

CrossRef (https://doi.org/10.1063/1.4984334)

Google Scholar (http://scholar.google.com/scholar_lookup? title=Full\%20parabolic\%20trough\%20qualification\%2ofrom\%2oprototype\%20 to\%20demonstration\%2oloop\%2C\%20Solarpaces\%202016\&author=N.\%2OJan otte\&author=E.\%20Luepfert\&author=K.\%20Pottler\&author=M.\%20Schmitz\&j ournal=AIP\%20Conference\%20Proceedings\&volume $=1850 \&$ pages $=020010 \& p$ ublication_year $=2017 \&$ doi $=10.1063 \% 2 \mathrm{~F} 1.4984334$ )

8. Lüpfert E, Pottler K, Riffelmann KJ, Ulmer S, Schiricke B, Neumann A (2007) Parabolic trough analysis techniques for optical performance. J Sol En Eng 129. https://doi.org/10.1115/1.2710249 (https://doi.org/10.1115/1.2710249)

9. Lüpfert E, Riffelmann K-J, Price H, Moss T (2008) Experimental analysis of overall thermal properties of parabolic trough receivers. J Sol Energy Eng 130:021007. https://doi.org/10.1115/1.2888756

(https://doi.org/10.1115/1.2888756)

CrossRef (https://doi.org/10.1115/1.2888756)

Google Scholar (http://scholar.google.com/scholar_lookup?

title=Experimental\%20analysis\%20of\%20overall\%20thermal\%2oproperties\%2 oof\%2oparabolic\%2otrough\%2oreceivers\&author=E.\%20L\%C3\%BCpfert\&auth or $=\mathrm{K}-$

J.\%20Riffelmann\&author $=$ H.\%20Price\&author $=$ T.\%20Moss\&journal=J\%20So $1 \% 20$ Energy $\% 20$ Eng\&volume $=130 \&$ pages $=021007 \&$ publication_year $=2008 \& d$ oi $=10.1115 \% 2 \mathrm{~F} 1.2888756)$

10. Marcotte P, Manning K (2014) Development of an advanced large-aperture parabolic trough collector, Energy Procedia 49:145-154.

https://doi.org/10.1016/j.egypro.2014.03.016

(https://doi.org/10.1016/j.egypro.2014.03.016)

11. Riffelmann KJ, Schweitzer A, Weinrebe G, Balz M (2019) Planning and building the first ultimate trough collector field in the Kingdom of Saudi Arabia. AIP Conference Proceedings 2126:040002. https://doi.org/10.1063/1.5117582 (https://doi.org/10.1063/1.5117582)

CrossRef (https://doi.org/10.1063/1.5117582)

Google Scholar (http://scholar.google.com/scholar_lookup?

title=Planning\%20and\%2obuilding\%20the\%2ofirst\%2oultimate\%2otrough\%2 ocollector\%2ofield\%20in\%2othe\%20Kingdom\%20of\%20Saudi\%20Arabia\&aut hor=KJ.\%20Riffelmann\&author=A.\%20Schweitzer\&author=G.\%20Weinrebe\& author $=$ M.\%2oBalz\&journal=AIP\%20Conference\%20Proceedings\&volume $=21$ 26\&pages $=040002 \&$ publication_year $=2019 \& d o \mathrm{i}=10.1063 \% 2 \mathrm{~F} 1.5117582)$

12. Schmitz M, et al. (2018) Heliotrough collector development.

www.heliotrough.com (http://www.heliotrough.com)

Google Scholar (http://scholar.google.com/scholar_lookup? title=Heliotrough\%20collector\%2odevelopment\&author=M.\%20Schmitz\&publ ication_year=2018) 
13. Schweitzer A, Schiel W, Birkle M, Nava P, Riffelmann KJ, Wohlfahrt A, Kuhlmann G (2014) ULTIMATE TROUGH - fabrication, erection and commissioning of the World's largest parabolic trough collector. Energy Procedia 49:1848-1857. https://doi.org/10.1016/j.egypro.2014.03.196 (https://doi.org/10.1016/j.egypro.2014.03.196)

CrossRef (https://doi.org/10.1016/j.egypro.2014.03.196)

Google Scholar (http://scholar.google.com/scholar_lookup? title=ULTIMATE\%2OTROUGH\%20-

\%2ofabrication\%2C\%20erection\%20and\%2ocommissioning\%20of\%2othe\%20 World\%27s\%2olargest\%2oparabolic\%20trough\%2ocollector\&author=A.\%20Sc hweitzer\&author=W.\%20Schiel\&author=M.\%20Birkle\&author=P.\%20Nava\&a uthor=KJ.\%20Riffelmann\&author=A.\%20Wohlfahrt\&author=G.\%20Kuhlman n\&journal=Energy\%20Procedia\&volume $=49$ \&pages $=1848$ -

$1857 \&$ publication_year $=2014 \&$ doi $=10.1016 \% 2 F j$.egypro.2014.03.196)

14. UNE (2018) https://www.une.org/encuentra-tu-norma/comites-tecnicos-denormalizacion/comite/?c=CTN\%20206/SC\%20117

(https://www.une.org/encuentra-tu-norma/comites-tecnicos-denormalizacion/comite/?c=CTN\%20206/SC\%20117)

\section{Books and Reviews}

www.abengoa.com (http://www.abengoa.com)

www.dlr.de/sf (http://www.dlr.de/sf) (technology, news, downloads, publications) www.estelasolar.eu (http://www.estelasolar.eu) (European CSP industry association) https://s3platform.jrc.ec.europa.eu/concentrated-solar-power (https://s3platform.jrc.ec.europa.eu/concentrated-solar-power) https://setis.ec.europa.eu/solar-thermal-electricity-concentrated-solar-powerimplementation (https://setis.ec.europa.eu/solar-thermal-electricity-concentratedsolar-power-implementation)

www.helioscsp.com (http://www.helioscsp.com) and www.protermosolar.com (http://www.protermosolar.com) (news and industry association) www.nrel.gov/csp (http://www.nrel.gov/csp) (technical info base, publications, projects list)

www.psa.es (http://www.psa.es) (Plataforma Solar de Almería, Spain)

www.rioglass.com (http://www.rioglass.com)

www.solarpaces.org (http://www.solarpaces.org) (International Energy Agency, CSP implementing agreement)

\section{Copyright information}

(C) Springer Science+Business Media, LLC, part of Springer Nature 2021

\section{Section editors and affiliations}

- Spiros Alexopoulos (1)

- Soteris Kalogirou (2) 
1. Solar-Institut Jülich (SIJ), FH Aachen. University of Applied Sciences, , Jülich, Germany

2. Department of Mechanical Engineering and Materials Sciences and Engineering, Cyprus University of Technology, , Limassol, Cyprus

\section{How to cite}

Cite this entry as:

Lüpfert E. (2021) Parabolic Trough Solar Technology. In: Meyers R.A. (eds) Encyclopedia of Sustainability Science and Technology. Springer, New York, NY. https://doi.org/10.1007/978-1-4939-2493-6_678-3

\section{About this entry}

- Received 20 July 2020

- Accepted 04 October 2020

- First Online 31 January 2021

- DOI https://doi.org/10.1007/978-1-4939-2493-6

- Publisher Name Springer, New York, NY

- Online ISBN 978-1-4939-2493-6

- eBook Packages Earth and Environmental Science Reference Module Physical and Materials Science

- $\underline{\text { Reprints and Permissions }}$

\section{SPRINGER NATURE}

(C) 2020 Springer Nature Switzerland AG. Part of $\underline{\text { Springer Nature. }}$

Not logged in Deutsches Zentrum für Luft \& Raumfahrt (3000132882) - Nationallizenz Zeitschriften-Archiv (3000137278) - Nationallizenz Ebooks Medicine (3000137282) - Zeitschriftenkons d Helmholtz Gemeinschaft vertreten durch das (3000175443) - Nationallizenz Lecture Notes-Archiv (3000262825) - Nationallizenz Ebooks Chemistry and Materials Science 2005-2008 (3000262839) - DEAL DE / Springer Compact Clearingstelle Uni Freiburg _ (3003169293) - MPDL Services GmbH c/o Max-Planck-Gesellschaft zur (3902044458) - Palgrave Connect - eBooks E+F - CY 2000- German National Library of Economics (3994313832) 129.247.247.240 